\title{
Colloidal PbS and PbSeS Quantum Dot Sensitized Solar Cells Prepared by Electrophoretic Deposition
}

Nima Parsi Benehkohal, ${ }^{* a}$ Victoria González-Pedro, ${ }^{\mathrm{b}}$ Pablo P. Boix, ${ }^{\mathrm{b}}$ Sudam Chavhan, ${ }^{\mathrm{c}}$ Ramón Tena-Zaera, ${ }^{\mathrm{c}}$ George P. Demopoulos, ${ }^{\mathrm{a}}$ and Iván Mora-Seró ${ }^{* \mathrm{~b}}$

a Department of Materials Engineering, McGill Universit, Montreal, QC H3A 2B2, (Canada)

${ }^{\mathrm{b}}$ Photovoltaic and Optoelectronic Devices Group

Departament de Física, Universitat Jaume I, 12071 Castelló de la Plana (Spain), E-mail: sero@fca.uji.es

c Energy Department, IK4-CIDETEC, Parque Tecnológico,Paseo Miramón 196, Donostia-San Sebastián, 20009, (Spain)

\begin{abstract}
:
Here we report the developement of quantum dot sensitized solar cells (QDSCs) using colloidal $\mathrm{PbS}$ and $\mathrm{PbSeS} \mathrm{QDs}$ and polysulfide electrolyte for high photocurrents. QDSCs have been prepared in a novel sensitizing way employing electrophoretic deposition (EPD), and protecting the colloidal QDs from corrosive electrolyte with a CdS coating. EPD allows a rapid, uniform and effective sensitization with QDs, while the CdS coating stabilizes the electrode. The effect of electrophoretic deposition time and of colloidal QD size on cell efficiency is analyzed. Efficiencies as high as $2.1 \pm 0.2 \%$ are reported.
\end{abstract}

Corresponding Authors: nima.parsibenehkohal@mail.mcgill.ca and sero@uji.es

Key words: PbS, PbSeS, Quantum dots, Electrophoresis, Solar Cells 


\section{Introduction}

The development of third generation solar cells overcoming the Shockley-Queisser efficiency limit for a single absorber, $31 \%,{ }^{1}$ is one of the most fascinating challenges in the energy research field. In this aspect, semiconductor Quantum Dots (QDs) have shown extremely attractive properties for the development of solar cells overcoming the current limitations. ${ }^{2}$ The demonstration of efficient multiple exciton generation (MEG) process in colloidal QDs, ${ }^{3,4}$ despite certain controversy, ${ }^{5}$ has aroused a huge interest in the use of these materials in photovoltaic devices. This interest has been reinforced with the recent reports of absorbed photon-to-current efficiency (APCE) close to $200 \%{ }^{6}$ and incident photon-to-current efficiency (IPCE) as high as $114 \% .^{7}$ These achievements are acquired by using QDs with IR absorption, $\mathrm{PbS}^{6}$ and $\mathrm{PbSe} .{ }^{7}$ In the former case $\mathrm{PbS}$ QDs have been employed in a sensitized solar cell configuration. ${ }^{8}$ Electron-hole pairs, photogenerated and produced by impact ionization in a MEG process, in PbS colloidal QDs are quickly separated into two different media. Electrons are injected into flat $\mathrm{TiO}_{2}$ single crystals while holes are regenerated by a polysulfide electrolyte. ${ }^{6}$ Nanostructured $\mathrm{TiO}_{2}$ electrodes, instead of flat electrodes, enhance dramatically light harvesting but two main problems have to be solved: i) the uniform sensitization with colloidal QDs of nanostructured electrode along all its thickness and ii) the development of a stable QDSC configuration with colloidal PbS QDs as sensitizers. We have addressed these problems preparing colloidal $\mathrm{PbS}$ and $\mathrm{PbSeS}$ quantum-dot sensitized solar cells (QDSCs) in a novel sensitizing way employing electrophoretic deposition (EPD) and protecting the colloidal QDs from corrosive electrolyte with a CdS coating. We have analyzed the effect of electrophoretic deposition time and QD size in the final solar cell performance, obtaining efficiencies as high as $2.1 \pm 0.2 \%$. These results represent a significant advance in the development of colloidal QDSCs with light absorption in the IR region. In addition, we discuss the role of QDs in the recombination process of the analyzed solar cells.

Electrophoretic deposition has been used for the deposition of $\mathrm{TiO}_{2}$ nanoparticles in solar cell ${ }^{9}$ or photocatalytic ${ }^{10}$ applications. In addition, it is a method also employed to deposit colloidal QDs, especially of CdSe on different materials such $\mathrm{Au},{ }^{11,}{ }^{12}$ patterned electrodes, ${ }^{13}$ stacked-cup carbon nanotubes, ${ }^{14}$ and polymer templates. ${ }^{15}$ Colloidal CdSe QDs have also been deposited by electrophoresis for 
photovoltaic purposes. Electrophoretic deposition of $\mathrm{CdSe}-\mathrm{C} 60$ was used for the preparation of composite films for solar energy generation. ${ }^{16}$ Flexible QDSCs have been fabricated by using the electrophoretic deposition of CdSe QDs on $\mathrm{ZnO}$ nanorods, obtaining efficiencies of $0.98 \% .{ }^{17}$ Higher efficiencies, $1.7 \%$, have been reported for $\mathrm{TiO}_{2}$ nanostructured electrodes with a $\mathrm{ZnS}$ coating of the colloidal CdSe QDs. ${ }^{18}$ But there is no report on the use of EPD of PbS or PbSeS QDs. Electrophoretic deposition presents a significant advantage over other deposition techniques for colloidal QDs, either as linker assisted ${ }^{19-21}$ or direct adsorbed, ${ }^{19,21}$ because of its simplicity and short deposition time. While for electrophoretic deposition times as short as $2 \mathrm{~h}$ were sufficient for effective coating, ${ }^{18}$ several hours or even days are needed with other techniques. ${ }^{19-21}$

The use of PbS QDs in QDSCs has been significantly less than the utilization of CdSe QDs, in spite of the higher light harvesting potential of PbS QDs due to their tunable absorption in the IR range. This is largely due to the difficulty of finding an appropriate electrolyte for $\mathrm{PbS}$ in which it is stable. $\mathrm{PbS}$ is not stable neither with iodine nor polysulfide redox electrolytes. ${ }^{22-24}$ Thus, most of the reports on PbS QDSCs are for all-solid devices. ${ }^{23,25-27}$ In the case of using a liquid electrolyte for hole transport in $\mathrm{PbS}$ QDSCs, the highest reported efficiency, 0.62\%, has been reported using a Co redox electrolyte, ${ }^{28}$ at 1 sun and with $\mathrm{PbS}$ grown by the Successive Ionic Layer Absorption and Reaction (SILAR) method. We have shown that by employing the same deposition technique, stable QDSCs using polysulfide electrolyte can be obtained by coating the $\mathrm{PbS}$ QDs with $\mathrm{CdS},{ }^{29}$ reporting a significant efficiency of $2.36 \%$ using nanostructured $\mathrm{TiO}_{2}$ electrodes. ${ }^{30}$ Similar efficiencies have been obtained using $\mathrm{SnO}_{2}$ electrodes, ${ }^{31}$ and outstanding efficiencies of $3.82 \%$ have been obtained using $\mathrm{TiO}_{2}$ photoanodes with hierarchical pore distribution, ${ }^{32}$ employing again in both cases the SILAR growth. But, the presynthesis of colloidal QDs allows the preparation of QDs with better defined properties than QD samples prepared by SILAR. Treatment of colloidal PbS/TiO2 cells using CdS grown bySILAR method has been successfully applied before in a depleted heterojunction solar cell configuration. ${ }^{33}$ The ultrafast electron injection from $\mathrm{PbS}$ colloidal QDs into $\mathrm{TiO}_{2}$ as fast as $6.4 \mathrm{fs},{ }^{34}$ points to the capability of extraction of charge generated by MEG. Thus the preparation of cells with colloidal QDs is extremely interesting.

\section{Experimental Section}


Colloidal QDs: PbSSe QDs with oleic acid capping were kindly provided by NANOCO, while PbS QDs were purchased from Evident Technologies. Both QDs were solved in toluene.

$\mathrm{TiO}_{2}$ photoanode Preparation: After cleaning the FTO glasses (Pilkington TEC 8 with 8 $\Omega^{2}$ sheet resistance), a compact layer of $\mathrm{TiO}_{2}$ was deposited on them by spray pyrolysis of titanium- (IV) bis(acetoacetonato) di(isopropanoxylate) followed by sintering at $450^{\circ} \mathrm{C}$, in order to improve the electrical contact between the nanoparticles. $\mathrm{TiO}_{2}$ photoanodes were prepared by "double-layer" screen-printing on FTO glass using two different $\mathrm{TiO}_{2}$ pastes including a light-scattering layer on top of the transparent $\mathrm{TiO}_{2}$ film. The transparent layer is formed by $20 \mathrm{~nm} \mathrm{TiO}{ }_{2}$ nanoparticles (18NR-AO, Dyesol) and the opaque layer contains 300-400 nm $\mathrm{TiO}_{2}$ Particles (WER4-O Dyesol). Finally, the resulting film was sintered again at $450{ }^{\circ} \mathrm{C}$ for 30 minutes. Total thickness of the photoanodes was $15 \pm 1 \mu \mathrm{m}$, measured with a profilometer Dektack 6 from Veeco.

Electrophoretic Deposition of the $Q D s$ on the $\mathrm{TiO}_{2}$ Electrodes: QDs were diluted in toluene, with concentrations of $\sim 2.2 \cdot 10^{-6} \mathrm{M}$. Two $\mathrm{TiO}_{2}$ FTO electrodes were immersed vertically in the QDs solution parallel to each other. The deposition area of the electrodes was about $0.25 \mathrm{~cm}^{2}$ and the distance between them was adjusted at $1 \mathrm{~cm}$. A voltage of $200 \mathrm{~V}$ was applied during 5-90 min. QDs were deposited on both cathode and anode electrodes similar to previous reports. ${ }^{18}$ Fresh layers at each deposition time were taken out from the electrophoretic cell, rinsed several times with toluene to wash off unbound QDs and subsequently rinsed with ethanol and dried at room temperature. After electrophoretic deposition colloidal QDs were coated with CdS layer grown by SILAR. The SILAR process has been carried out following the method recently described. $\mathrm{Cd}^{2+}$ ions have been deposited from an ethanolic $0.05 \mathrm{M}$ solution of $\mathrm{Cd}\left(\mathrm{NO}_{3}\right)_{2} \times 4 \mathrm{H}_{2} \mathrm{O}$. The sulfide sources were a $0.05 \mathrm{M}$ solutions of $\mathrm{Na}_{2} \mathrm{~S} \times 9 \mathrm{H}_{2} \mathrm{O}$ in methanol/water $(50 / 50 \mathrm{~V} / \mathrm{V})$. A single SILAR cycle consisted of 1 minute dip-coating of the $\mathrm{TiO}_{2}$ working electrode into the metal precursors and subsequently rinsed during one minute in ethanol. Subsequently sample is dipped into the sulfide solutions for one minute and rinsed in methanol/water $(50 / 50 \mathrm{~V} / \mathrm{V})$ one more minute. This procedure constitutes a complete SILAR cycle. SILAR process has been carried out automatically using a robot designed by ISTest. All the analyzed cells in this work were coated with $\mathrm{ZnS}$, by being alternately dipped into $0.1 \mathrm{M} \mathrm{Zn}\left(\mathrm{CH}_{3} \mathrm{COO}\right)_{2}$ and $0.1 \mathrm{M} \mathrm{Na} 2 \mathrm{~S}$ Milli-Q water solutions for $1 \mathrm{~min} / \mathrm{dip}$ and subsequently rinsed with Milli-Q ultrapure water. Two SILAR cycles were employed for $\mathrm{ZnS}$ coating. 
QDSC Preparation: Porous $\mathrm{Cu}_{2} \mathrm{~S}$ was used as counter-electrodes which was prepared by immersing brass in $\mathrm{HCl}$ solution at $70^{\circ} \mathrm{C}$ for $5 \mathrm{~min}$ and subsequently dipping it into polysulfide solution for $10 \mathrm{~min} .{ }^{19}$ The counter-electrode and a QD-sensitized electrode were assembled into sandwich type the using a scotch spacer (thickness $50 \mu \mathrm{m}$ ) and with a droplet $(10 \mu \mathrm{l})$ of polysulfide electrolyte. Polysulfide electrolyte was composed of $1 \mathrm{M} \mathrm{Na}_{2} \mathrm{~S}, 1 \mathrm{M} \mathrm{S}$, and $0.1 \mathrm{M} \mathrm{NaOH}$ solution in Milli-Q ultrapure water.

Photoanode and Solar Cell Characterization: The cross section morphology of the $\mathrm{TiO}_{2}-\mathrm{PbSeS}$ electrode films was investigated using a field emission scanning electron microscope FE-SEM (ULTRA plus ZEISS FESEM). Energy-dispersive X-ray spectroscopy (Apollo X, Ametek® EDAX) was employed to map and determine the distribution of chemical elements. A Bruker AXS-D8 Advance X-ray diffractometer (XRD), using $\mathrm{Cu} \mathrm{K} \alpha$ radiation, was used to analyze the structural properties of anodes before and after light sensitization. The optical absorption spectra of the photoanodes were recorded at 300-700 nm using a Cary 500 UV vis Varian photospectroscometer. The IPCE measurements were done using a $150 \mathrm{~W}$ Xe lamp coupled with a monochromator controlled by a computer; the photocurrent was measured using an optical power meter 70310 from Oriel Instruments, using a Si photodiode to calibrate the system. QDSCs were characterized by current-voltage and impedance spectroscopy using a $0.1256 \mathrm{~cm}^{2}$ mask and no antireflective layer. These measurements were performed employing the PG-STAT30 potentiostat (Autolab) and solar simulator at AM1.5 G, where the light intensity was adjusted with an NREL calibrated Si solar cell with a KG-5 filter to one sun intensity $\left(100 \mathrm{~mW} / \mathrm{cm}^{2}\right)$. For most of the conditions analyzed in this work more than one cell have been prepared, standard errors have been calculated for these conditions, and included in Tables 1 and 2. In few cases just a single cell was analyzed, in that cases errors are not provided. IS measurements were carried out in dark at different bias voltages with $10 \mathrm{mV}$ AC perturbation over a frequency range of $400 \mathrm{kHz}$ to $10 \mathrm{mHz}$.

\section{Results and Discussion}

QDSCs have been prepared in this work by electrophoretic deposition of $\mathrm{PbS}$ and $\mathrm{PbSeS}$ QDs. It has been shown that PbSeS QDs offer certain benefits with respect to $\mathrm{PbS}$ or $\mathrm{PbSe} \mathrm{QDs}$ in depleted heterojunction solar cells. ${ }^{35}$ Figure 1 , shows a cross section of a PbSeS sensitized $\mathrm{TiO}_{2}$ film prepared by EPD. The double layer structure of $\mathrm{TiO}_{2}$ can be appreciated with a thicker $\mathrm{TiO}_{2}$ transparent layer and a thinner top 
scattering layer. Elementary mapping of $\mathrm{Ti}, \mathrm{O}, \mathrm{Se}, \mathrm{S}$ and $\mathrm{Pb}$ at three different positions (i.e. sample depths) indicate that the deposition of colloidal PbSeS QDs was uniform along $\mathrm{TiO}_{2}$ thickness, ruling out a preferential deposition on top layer. A rather continuous coating, with no inhomogeneities at nanoscale level, is detected by comparing high magnification SEM micrographs of samples before and after electrophoretic deposition (Figure S1). It is worth to mention that no effect of the electrophoretic deposition time, $t_{d}$, on the coating homogeneity was detected.

Solar cells prepared with photoanodes sensitized with $\mathrm{PbS}$ and $\mathrm{PbSeS}$ QDs show poor stability with polysulfide electrolyte. It has been shown that $\mathrm{PbS}$ is photocorroded in polysulfide electrolyte, ${ }^{23}$ and needs to be protected from direct contact with the electrolyte. This is accomplished in this work by coating of CdS layer, using SILAR technique, on top of $\mathrm{PbSeS}$ and $\mathrm{PbS}$ QDs deposited by electrophoresis. CdS coating has been demonstrated previously to be an efficient protection of $\mathrm{PbS}$, with a significant enhancement of the solar cell efficiency and stability. ${ }^{29}$ In this sense, we have used a $\mathrm{CdS}$ coating, deposited by SILAR, in order to protect the PbSeS and PbS QDs from the corrosive effect of polysulfide electrolyte, obtaining stable devices.

For photoanode sensitization with $\mathrm{PbSeS} 800 \mathrm{~nm}$ different deposition times $\left(t_{d}\right)$ have been investigated. Hereafter, to distinguish among the different QD sizes analyzed after the QD type ( $\mathrm{PbS}$ or $\mathrm{PbSeS})$ we will add the wavelength of the first excitonic absorption peak. Figure $2 \mathrm{a}$ presents the absorption, in the allowed range of our experimental setup, of differently sensitized $\mathrm{TiO}_{2}$ films: bare $\mathrm{TiO}_{2}$ film, film sensitized just with 5 SILAR cycles of CdS, films sensitized with $800 \mathrm{~nm} \mathrm{PbSeS}$ QDs plus 5 SILAR cycles of CdS at different deposition times. The absorption of the different samples has been extracted from their diffuse reflectance $R$ and it is expressed in Kubelka-Munk units as $F(R)=(1-R)^{2} / 2 R$. For the sensitized electrodes the absorption of $\mathrm{TiO}_{2}$ substrate has been removed. The film sensitized just with $\mathrm{CdS}$ exhibits an absorption threshold at $550 \mathrm{~nm}$. When $\mathrm{PbSeS}$ QDs are deposited and coated with $\mathrm{CdS}$ the absorption threshold red shifts causing light absorption in the red visible region to increase due to a higher colloidal QD loading with deposition time.

Figure 2b, shows the current-potential curves obtained for QDSCs using photoanodes with different $t_{d}$, the solar cell parameters corresponding to these cells can be found in Table 1. When colloidal PbSeS QDs are deposited before CdS, the photocurrent of the cells increases due to the higher light harvesting capability provided by $\mathrm{PbSeS}$ QDs, see Figure 2a. But, this is associated with a decrease in open circuit 
voltage, $V_{o c}$, as the QD loading increases. It should be expected that the ligands are preserved in the electrophoretic deposition, although the photoinjection is still possible. Efficient photoinjection has been previously reported for devices using colloidal QDs. CdSe QDs capped with TOP directly adsorbed on $\mathrm{TiO}_{2}$ showing an APCE rather high, $\sim 90 \%,{ }^{36}$ and also colloidal QDS attached to $\mathrm{TiO}_{2}$ using linker molecules present significant photocurrent. ${ }^{19,21,37}$

The maximum performance in the analyzed cases has been obtained for $t_{d}=60$ min. The origin of the decrease of $V_{o c}$ can be understood by the analysis of impedance spectroscopy (EIS) data on the basis of the previously proposed models. ${ }^{38,} 39$ Samples with different deposition time present the same chemical capacitance, see Supporting Information S2, indicating that the conduction band position and the density of states of $\mathrm{TiO}_{2}$ are not affected by the QD loading. A clear trend in the recombination resistance is observed, Figure 2c. The recombination resistance is seen to decrease with $t_{d}$, indicating unambiguously that $\mathrm{PbSeS}$ QDs participate in the recombination process. ${ }^{40}$ Recombination increases with the QD loading. PbSeS QDs are acting as recombination centers as it has been also observed recently for $\mathrm{Sb}_{2} \mathrm{~S}_{3}{ }^{41,}{ }^{42}$ The observation of this fact is decisive for the future optimization of the QDSCs. On the other hand, the reduction of recombination resistance due to increase in QD loading produces the observed decrease in $V_{o c}$, which adversely affect on the solar cell performance, see Table 1 . Note that both $\mathrm{PbSeS}$ colloidal QDs and $\mathrm{CdS}$ coating contribute to the final light harvesting. PbSeS QDs are the responsible of light absorption for wavelengths lower than $\sim 550 \mathrm{~nm}$, while for wavelengths higher than $\sim 550 \mathrm{~nm}$ both PbSeS QDs and CdS contribute to the light absorption, but with a higher part from CdS, see Figure 2a.

We have also analyzed the effect of QD size in the final solar cell performance using $\mathrm{PbSeS}$ and $\mathrm{PbS}$ QDs of different sizes keeping $t_{d}$ constant, see Figure 3. As the first excitonic absorption peak shifts to the IR region, the light absorption in the visible region increases, Figure 3a. But the increase in the light harvesting capability does not translate into greater efficiency of QDSCs, or in an increase of the photocurrent, $J_{s c}$, see Figure $3 \mathrm{~b}$ and Table 2. In fact, a systematic decrease of $V_{o c}$ and $J_{s c}$ is observed, with the highest efficiency obtained using PbS $743 \mathrm{~nm}$ QDs, i.e. the QDs with the smallest size (largest band gap). The relation between the wavelength of the first excitonic absortion peak and $\mathrm{PbS}$ QD size is described in Supporting Information S3, using data from reference $^{43}$. 
The decrease of solar cell performance with the increase of the size of QDs has two causes. On one hand, the recombination resistance depends on the QD size, see Figure 3d. The sample with $\mathrm{PbS} 743 \mathrm{~nm}$ presents the highest recombination resistance (lowest recombination rate). On the other hand, there is an especially interesting discrepancy between light absorption and incident photon to current efficiency (IPCE), Figure 3c. This discrepancy is clearly manifested for the biggest QDs, PbS $1427 \mathrm{~nm}$. In this case, the sensitized photoanode presents strong light absorption in all the visible region, but practically null IPCE at wavelengths higher than $500 \mathrm{~nm}$. Indicating that $\mathrm{PbS}$ is not contributing to the photocurrent. In this case only $\mathrm{CdS}$ light absorber is contributing to the photocurrent, as can be observed from the IPCE measurements. This result is in good agreement with the work of Hyun et al. ${ }^{43}$ In that work it has been shown that $\mathrm{PbS}$ QDs with size bigger than $4.3 \mathrm{~nm}$ (wavelength of the first excitonic absorption peak: $1116 \mathrm{~nm}$ ) cannot inject into $\mathrm{TiO}_{2}$ conduction band (CB) as its conduction band is lower than the $\mathrm{CB}$ of $\mathrm{TiO}_{2}$, as it is indicated schematically in Figure 3e. As the QD size decreases the band gap increases shifting the $\mathrm{PbS} \mathrm{CB}$ to higher energies than the $\mathrm{TiO}_{2} \mathrm{CB}$, allowing electron injection from $\mathrm{PbS}$ with small size into $\mathrm{TiO}_{2}$. As the quantum confinement increases the energetic distance between both CBs increases too, enhancing the injection driving force and consequently the photocurrent.

Having shown that the recombination pathway is preferentially through $\mathrm{PbS}$ QDs, and also that depends on the QDs size, recombination in this QDSCs has to be related with QD traps. Note that for $\mathrm{PbS} 1427 \mathrm{~nm}$ QDs, that do not inject electrons into $\mathrm{TiO}_{2}$, the cell performance is significantly lower than for the cell just with $5 \mathrm{CdS}$ SILAR cycles, see Figure $3 b$. This implies that the PbS QDs act as recombination centers in all the analyzed cases even when they are not able to inject photoexcited electrons into $\mathrm{TiO}_{2}$. On the other hand, PbSeS QDs present lower recombination resistance than $\mathrm{PbS}$ indicating a higher recombination rate than their $\mathrm{PbS}$ counterparts.

Additionally, for the QD size with the highest performance, PbS $743 \mathrm{~nm}$, we have modified the number of CdS SILAR cycles obtaining an efficiency as high as $2.1 \pm 0.2 \%$ for 9 SILAR cycles $(1.8 \%$ for the sample just with 9 SILAR cycles of CdS), see Figure $3 b$ and Table 2 . Significantly this efficiency is very close to our previously reported efficiency of $2.21 \%$ for a $\mathrm{PbS} / \mathrm{CdS}$, both grown by SILAR and using the same $\mathrm{TiO}_{2}$ electrode, ${ }^{29}$ that conventionally produces solar cells with higher efficiencies than colloidal QDs. ${ }^{44}$ 


\section{Conclusions}

In summary, we have sensitized nanostructured $\mathrm{TiO}_{2}$ photoanodes with colloidal QDs of $\mathrm{PbSeS}$ and $\mathrm{PbS}$ with different sizes. We have shown that the electrophoretic deposition method can used advantageously for fast sensitization of the photoanode with these QDs. CdS coating, deposited by SILAR, protects the colloidal QDs stabilizing the solar cell performance. A clear effect between QD size and device performance is observed, obtaining better results for the smallest QDs, with efficiencies as high as $2.1 \pm 0.2 \%$. In addition, we have shown unambiguously that QDs act as recombination centers in these QDSCs. There is plenty of room for the optimization of these devices by focusing in reducing recombination though the QD traps. The latter may be possible by improving control on the QD properties, further characterization and surface treatments seem thus to be crucial. As an example, PbSeS sensitized photoanodes were here characterized by X-ray diffraction and the presence of lead oxide $(\mathrm{PbO})$ phase was detected, irrespectively of the $t_{d}$, (Supporting Information $\mathrm{S} 4$ ). Although the origin of oxidation and its final effect in solar cell performance is currently under investigation, this finding points out the wide room of improvement of present lead chalcogenide QDSCs.

\section{Associated Content}

Supporting Information. FE-SEM micrographs of cross sections of $\mathrm{TiO}_{2}$ and $\mathrm{TiO}_{2} / \mathrm{PbSSe}$ samples, Chemical Capacitance of the analyzed cells, Correlation between $\mathrm{PbS}$ QD size and wavelength of the first excitonic absorption peak, XRD Characterization. This material is available free of charge via the Internet at http://pubs.acs.org.

\section{Authors Information}

Corresponding Authors

*E-mail: nima.parsibenehkohal@mail.mcgill.ca and sero@uji.es.

\section{Acknowledgement}

The work is supported by the "Institute of Nanotechnologies for Clean Energies", funded by the Generalitat Valenciana under project ISIC/2012/008. This work was partially supported by the European Union under the project ORION CP-IP 229036-2, the Ministerio de Educación y Ciencia of Spain under the project HOPE CSD2007- 
00007 (Consolider-Ingenio 2010) and JES-NANOSOLAR PLE2009-0042, by Generalitat Valenciana under project PROMETEO/2009/058, NSERC (Canada) strategic project grant and by McGill University MEDA scholarship. R.T-Z. acknowledges the support of the Program "Ramón y Cajal" of the MICINN. We acknowledge NANOCO for providing kindly PbSeS QDs.

\section{References}

1. W. Shockley and H. J. Queisser, J. Appl. Phys., 1961, 32, 510-519.

2. A. J. Nozik, Physica E, 2002, 14, 115-200.

3. R. D. Schaller and V. I. Klimov, Phys. Rev. Lett., 2004, 92, 186601.

4. R. J. Ellingson, M. C. Beard, J. C. Johnson, P. Yu, O. I. Micic, A. J. Nozik, A. Shabaev and A. L. Efros, Nano Lett., 2005, 5, 865-871.

5. M. T. Trinh, A. J. Houtepen, J. M. Schins, T. Hanrath, J. Piris, W. Knulst, A. P. L. M. Goossens and L. D. A. Siebbeles, Nano Lett., 2008, 8, 1713-1718.

6. J. B. Sambur, T. Novet and B. A. Parkinson, Science, 2010, 330, 63-66.

7. O. E. Semonin, J. M. Luther, S. Choi, H.-Y. Chen, J. Gao, A. J. Nozik and M. C. Beard, Science, 2011, 334, 1530-1533.

8. $\quad$ B. O' Regan and M. Grätzel, Nature, 1991, 353, 737-740.

9. $\quad$ L. Grinis, S. Dor, A. Ofir and A. Zaban, J. Photoch. Photobio. A, 2008, 198, 5259.

10. I. Mora-Seró, T. Lana-Villarreal, J. Bisquert, A. Pitarch, R. Gómez and P. Salvador, J. Phys. Chem. B, 2005, 109, 3371-3380.

11. M. A. Islam, Y. Xia, D. A. Telesca Jr., M. L. Steigerwald and I. P. Herman, Chem. Mater., 2004, 16, 49-54.

12. S. Jia, S. Banerjee and I. P. Herman, J. Phys. Chem. C, 2008, 112, 162-171.

13. M. A. Islam and I. P. Herman, Appl. Phys. Lett., 2002, 80, 3823-3825.

14. B. Farrow and P. V. Kamat, J. Am. Chem. Soc., 2009, 131, 11124-11131.

15. Q. Zhang, T. Xu, D. Butterfield, M. J. Misner, D. J. Ryu, T. Emrick and T. P. Russell, Nano Lett., 2005, 5, 357-361.

16. P. Brown and P. V. Kamat, J. Am. Chem. Soc., 2008, 130, 8890-8891.

17. J. Chen, W. Lei, C. Li, Y. Zhang, Y. Cui, B. Wang and W. Deng, Phys. Chem. Chem. Phys., 2011, 13, 13182-13184.

18. A. Salant, M. Shalom, I. Hod, A. Faust, A. Zaban and U. Banin, ACS Nano, 2010, 4, 5962-5968.

19. S. Giménez, I. Mora-Seró, L. Macor, N. Guijarro, T. Lana-Villarreal, R. Gómez, L. J. Diguna, Q. Shen, T. Toyoda and J. Bisquert, Nanotechnology, 2009, 20, 295204.

20. I. Robel, V. Subramanian, M. Kuno and P. V. Kamat, J. Am. Chem. Soc., 2006, $128,2385-2393$.

21. I. Mora-Seró, S. Giménez, F. Fabregat-Santiago, R. Gómez, Q. Shen, T. Toyoda and J. Bisquert, Accounts Chem. Res., 2009, 42, 1848-1857.

22. G. Hodes, J. Phys. Chem. C, 2008, 112, 17778-17787.

23. H. J. Lee, H. C. Leventis, S.-J. Moon, P. Chen, S. Ito, S. A. Haque, T. Torres, F. Nüesch, T. Geiger, S. M. Zakeeruddin, et al., Adv. Funct. Mater., 2009, 19, 2735-2742.

24. B. Ma, L. Wang, H. Dong, R. Gao, Y. Geng, Y. Zhu and Y. Qiu, Phys. Chem. Chem. Phys., 2011, 13, 2656-2658. 
25. H. J. Snaith, A. Stavrinadis, P. Docampo and A. A. W. Watt, Solar Ene., 2011, 85, 1283-1290.

26. K. P. Acharya, E. Khon, T. O'Conner, I. Nemitz, A. Klinkova, R. S. Khnayzer, P. Anzenbacher and M. Zamkov, ACS Nano, 2011, 5, 4953-4964.

27. S. H. Im, H.-J. Kim, S. W. Kim, S.-W. Kimb and S. I. Seok, Energ. Environ. Sci., 2011, 4, 4181-4186.

28. H. J. Lee, P. Chen, S.-J. Moon, F. Sauvage, K. Sivula, T. Bessho, D. R. Gamelin, P. Comte, S. M. Zakeeruddin, S. I. Seok, et al., Langmuir, 2009, 25, 7602-7608.

29. A. Braga, S. Giménez, I. Concina, A. Vomiero and I. Mora-Seró, J. Phys. Chem. Lett., 2011, 2, 454-460.

30. M. Samadpour, P. P. Boix, S. Giménez, A. Iraji Zad, N. Taghavinia, I. MoraSeró and J. Bisquert, J. Phys. Chem. C, 2011, 115, 14400-14407.

31. M. A. Hossain, Z. Y. Koh and Q. Wang, Phys. Chem. Chem. Phys., 2012, 14, 7367-7374.

32. N. Zhou, G. Chen, X. Zhang, L. Cheng, Y. Luo, D. Li and Q. Meng, Electrochem. Comm., 2012, 20, 97-100.

33. E. Kinder, P. Moroz, G. Diederich, A. Johnson, M. Kirsanova, A. Nemchinov, T. O'Connor, D. Roth and M. Zamkov, J. Am. Chem. Soc., 2011, 133, 2048820499.

34. Y. Yang, W. Rodríguez-Córdoba, X. Xiang and T. Lian, Nano Lett., 2012, 12, 303-309.

35. W. Ma, J. M. Luther, H. Zheng, Y. Wu and A. P. Alivisatos, Nano Lett., 2009, 9, 1699-1703.

36. S. Giménez, X. Xu, T. Lana-Villarreal, R. Gómez, S. Agouram, Muñoz-Sanjosé and I. Mora-Seró, J. Appl. Phys., 2010, 108, 064310.

37. D. F. Watson, J. Phys. Chem. Lett., 2010, 1, 2299-2309.

38. F. Fabregat-Santiago, G. Garcia-Belmonte, I. Mora-Seró and J. Bisquert, Phys. Chem. Chem. Phys., 2011, 13, 9083-9118.

39. V. Gónzalez-Pedro, X. Xu, I. Mora-Seró and J. Bisquert ACS Nano, 2010, 4, 5783-5790.

40. Hod, V. González-Pedro, Z. Tachan, F. Fabregat-Santiago, I. Mora-Seró, J. Bisquert and A. Zaban, J. Phys. Chem. Lett., 2011, 2, 3032-3035.

41. P. P. Boix, G. Larramona, A. Jacob, B. Delatouche, I. Mora-Seró and J. Bisquert, J. Phys. Chem. C, 2012, 116, 1579-1587.

42. P. P. Boix, Y. H. Lee, F. Fabregat-Santiago, S. H. Im, I. Mora-Seró, J. Bisquert and S. I. Seok, ACS Nano, 2012, 6, 873-880.

43. B.-R. Hyun, Y.-W. Zhong, A. C. Bartnik, L. Sun, H. D. Abruña, F. W. Wise, J. D. Goodreau, J. R. Matthews, T. M. Leslie and N. F. Borrelli, ACS Nano, 2008, 2, 2206-2212.

44. N. Guijarro, T. Lana-Villarreal, Q. Shen, T. Toyoda and R. Gómez, J. Phys. Chem. C, 2010, 114, 21928-21937. 


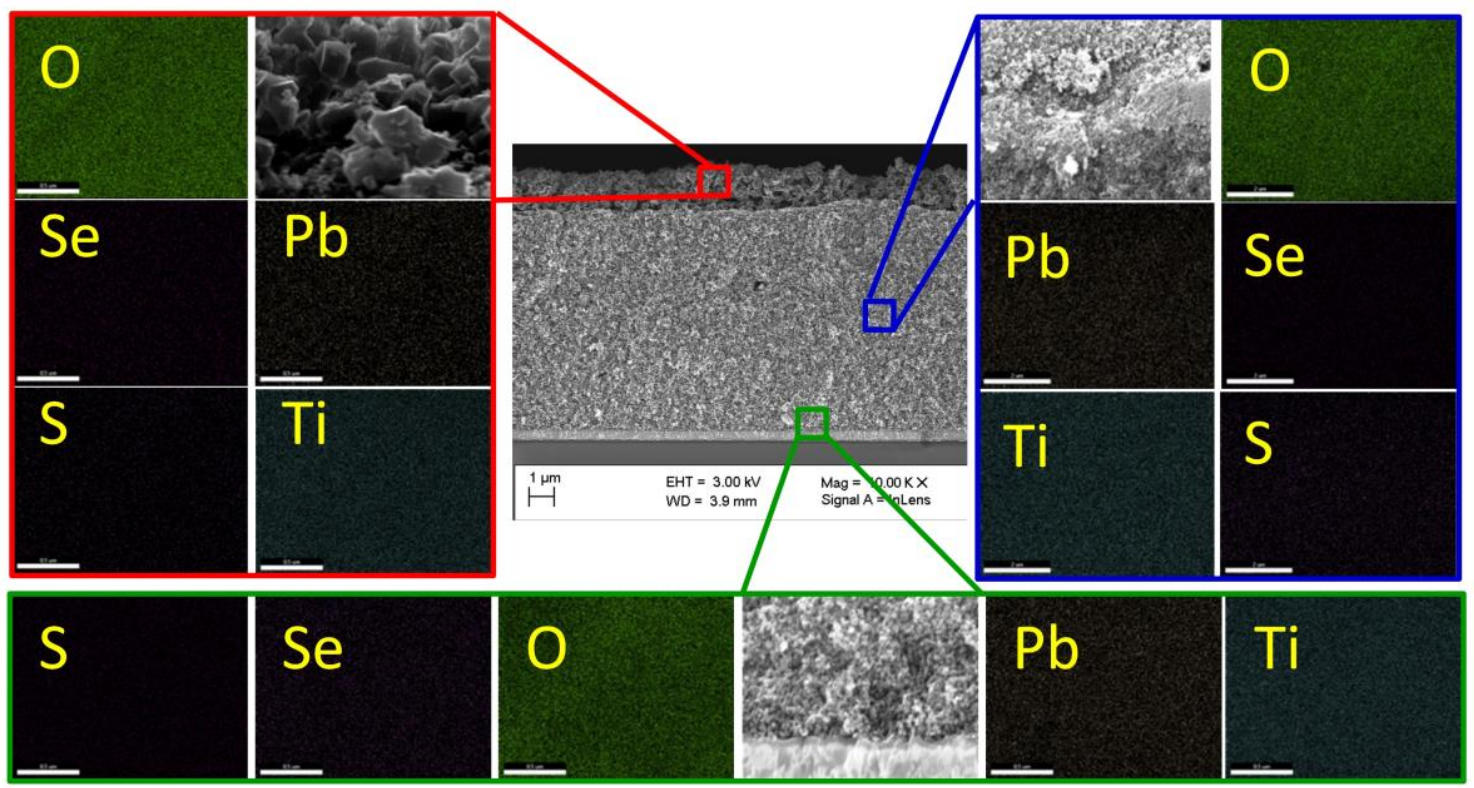

Figure 1. $\mathrm{SEM}$ analysis of a nanostructured $\mathrm{TiO}_{2}$ sensitized with $\mathrm{PbSeS} \mathrm{QDs}, t_{d}=60$ min. The central picture of a photoanode cross section is surrounded by a magnified image and elemental maps of the three square boxes in the central picture. Elemental maps display $\mathrm{Ti}, \mathrm{O}, \mathrm{Pb}, \mathrm{Se}$ and $\mathrm{S}$ spatial elemental distribution. 

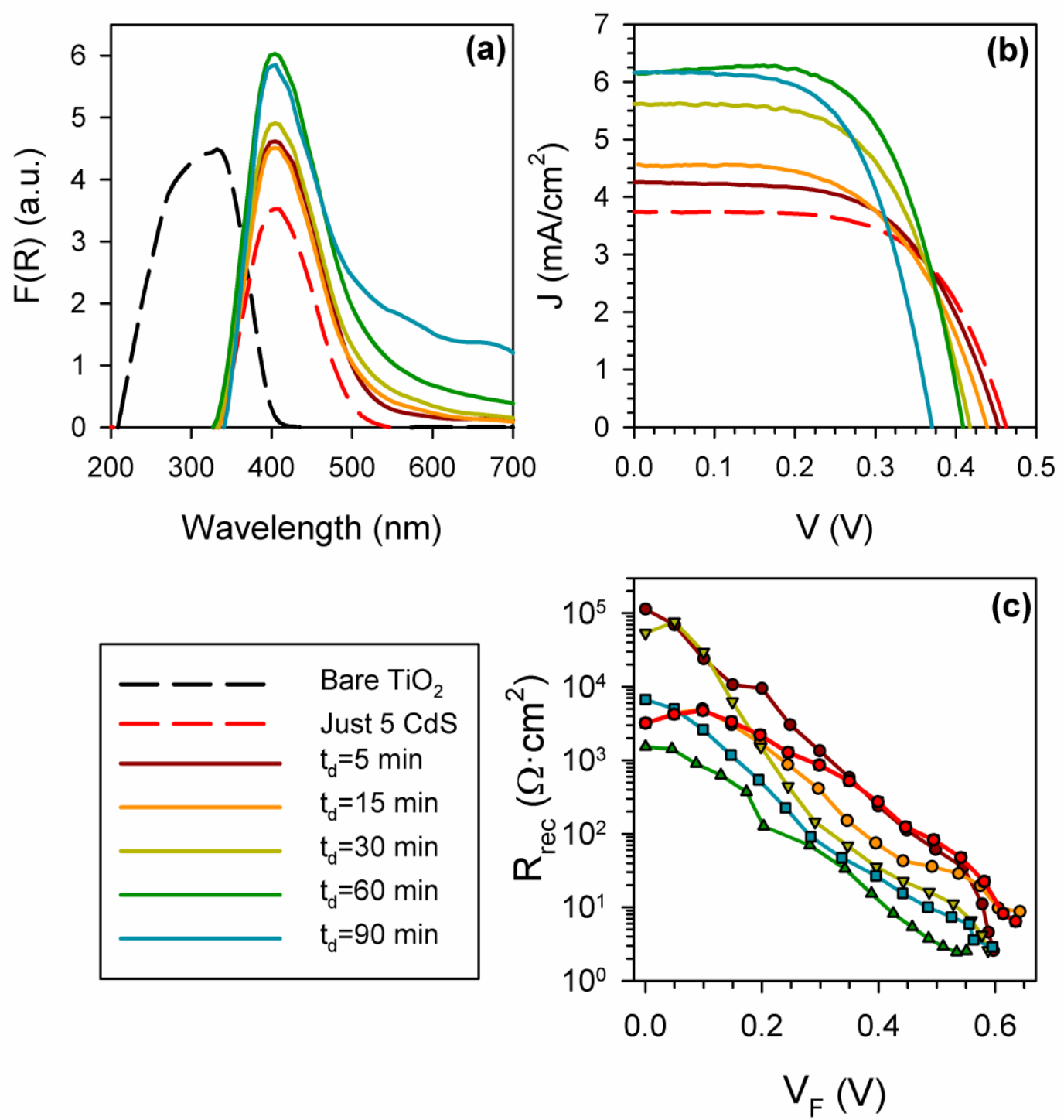

Figure 2. Effect of the electrophoretic deposition time, using $800 \mathrm{~nm} \mathrm{PbSeS}$ QDs, in a) Kubelka-Munk plot of the diffuse reflectance spectra for bare $\mathrm{TiO}_{2}$ film and $\mathrm{TiO}_{2}$ sensitized just with 5 SILAR cycles of CdS and with $800 \mathrm{~nm}$ PbSeS QDs plus 5 SILAR cycles of $\mathrm{CdS}$; in b) $\mathrm{J}-\mathrm{V}$ curve and in c) recombination resistance. 

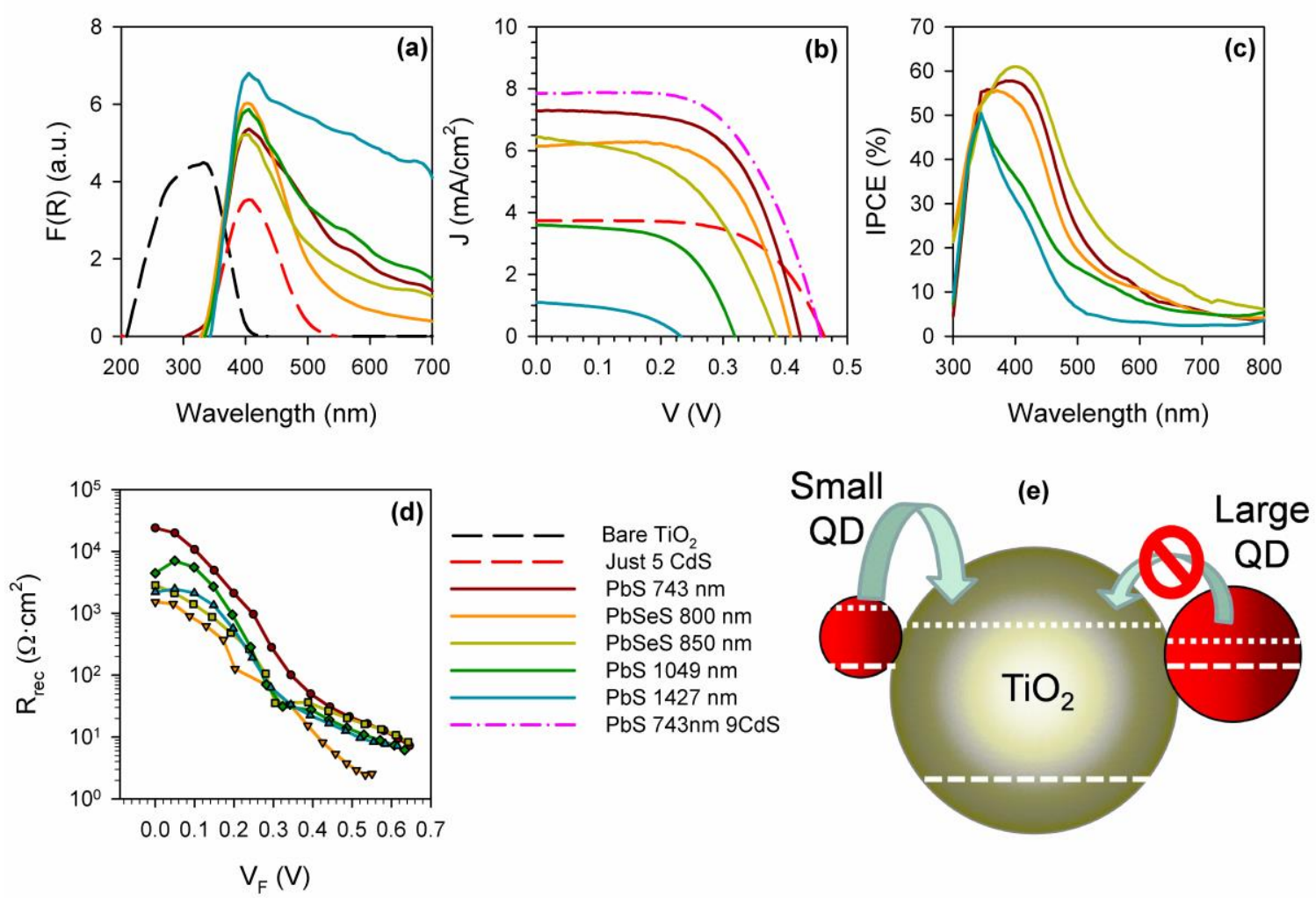

Figure 3. Effect of the QDs using the same electrophoretic deposition time, $60 \mathrm{~min}$, in a) Kubelka-Munk plot of the diffuse reflectance spectra for bare $\mathrm{TiO}_{2}$ film and $\mathrm{TiO}_{2}$ sensitized just with 5 SILAR cycles of CdS and with $800 \mathrm{~nm}$ PbSeS QDs plus 5 SILAR cycles of $\mathrm{CdS}$; in b) J-V curve; in c) IPCE; and in d) recombination resistance. e) Squeme of the relative alignment of the conduction band (dotted lines) of $\mathrm{TiO}_{2}$ and $\mathrm{PbS} / \mathrm{PbSeS} \mathrm{QDs}$ depending on the QD size, relative Valence bands (dashed lines) are also included as reference. 


\section{Tables:}

Table 1. Solar cell parameters of QDSCs prepared employing PbSeS $800 \mathrm{~nm}$ QDs and different electrophoresis deposition time. Open circuit voltage, $V_{o c}$, short circuit current, $J_{s c}$, fill factor, FF, and photovoltaic conversion efficiency, $\eta$.

\begin{tabular}{lllll}
\hline $\begin{array}{l}\mathrm{PbSeS} \\
800\end{array}$ & $\begin{array}{l}V_{o c} \\
(\mathrm{~V})\end{array}$ & $\begin{array}{l}J_{s c} \\
\left(\mathrm{~mA} / \mathrm{cm}^{2}\right)\end{array}$ & $\mathrm{FF}$ & $\begin{array}{l}\eta \\
(\%)\end{array}$ \\
$\mathrm{nm}^{[\mathrm{a}]}$ & & & & \\
\hline $5 \mathrm{CdS}$ & 0.46 & 3.7 & 0.62 & 1.07 \\
$5 \mathrm{~min}$ & $0.46 \pm 0.04$ & $4.3 \pm 0.5$ & $0.58 \pm 0.03$ & $1.14 \pm 0.14$ \\
$15 \mathrm{~min}$ & $0.440 \pm 0.15$ & $5 \pm 1$ & $0.56 \pm 0.01$ & $1.1 \pm 0.3$ \\
$30 \mathrm{~min}$ & $0.42 \pm 0.2$ & $5.6 \pm 0.6$ & $0.593 \pm 0.005$ & $1.4 \pm 0.2$ \\
$60 \mathrm{~min}$ & $0.41 \pm 0.2$ & $6.14 \pm 0.5$ & $0.63 \pm 0.01$ & $1.58 \pm 0.16$ \\
$90 \mathrm{~min}$ & 0.37 & 6.2 & 0.60 & 1.36
\end{tabular}

[a] 5CdS is a sample prepared with no PbSeS QDs and just 5 SILAR cycles of CdS and 2 SILAR cycles of $\mathrm{ZnS}$. The rest of the samples are identified by the $\mathrm{PbSeS}$ electrophoresis deposition time, in addition all these samples have also been coated with 5 SILAR cycles of CdS and 2 SILAR cycles of $\mathrm{ZnS}$.

Table 2. Solar cell parameters of QDSCs prepared employing QDs of different type and size. All the samples present the same electrophoresis deposition time, $60 \mathrm{~min}$, and CdS/ZnS coating, 5 and 2 SILAR cycles respectively, except the last one with 9 and 2 SILAR cycles. Sample prepared just with 9 SILAR cycles of CdS is included for comparison. Open circuit voltage, $V_{o c}$, short circuit current, $J_{s c}$, fill factor, FF, and photovoltaic conversion efficiency, $\eta$.

\begin{tabular}{lllll}
\hline$t_{d}=60 \mathrm{~min}$ & $\begin{array}{l}V_{o c} \\
(\mathrm{~V})\end{array}$ & $\begin{array}{l}J_{s c} \\
\left(\mathrm{~mA} / \mathrm{cm}^{2}\right)\end{array}$ & $\mathrm{FF}$ & $\begin{array}{l}\eta \\
(\%)\end{array}$ \\
\hline $\mathrm{PbS} \mathrm{743} \mathrm{nm}$ & $0.425 \pm 0.15$ & $7.3 \pm 0.7$ & $0.61 \pm 0.03$ & $1.9 \pm 0.2$ \\
$\mathrm{PbSeS} 800 \mathrm{~nm}$ & $0.41 \pm 0.2$ & $6.14 \pm 0.5$ & $0.63 \pm 0.01$ & $1.58 \pm 0.16$ \\
$\mathrm{PbSeS} 850 \mathrm{~nm}$ & 0.39 & 6.4 & 0.49 & 1.2 \\
$\mathrm{PbS} \mathrm{1049nm}$ & $0.322 \pm 0.015$ & $3.6 \pm 0.8$ & $0.584 \pm 0.014$ & $0.67 \pm 0.14$ \\
$\mathrm{PbS} \mathrm{1427nm}$ & $0.234 \pm 0.010$ & $1.09 \pm 0.12$ & $0.46 \pm 0.02$ & $0.1235 \pm 0.0003$ \\
\hline $\mathrm{PbS} \mathrm{743} \mathrm{nm}$ & $0.46 \pm 0.07$ & $8 \pm 2$ & $0.58 \pm 0.03$ & $2.1 \pm 0.2$
\end{tabular}


9CdS

9CdS

0.515

5.79

0.60

1.8

TOC figure:

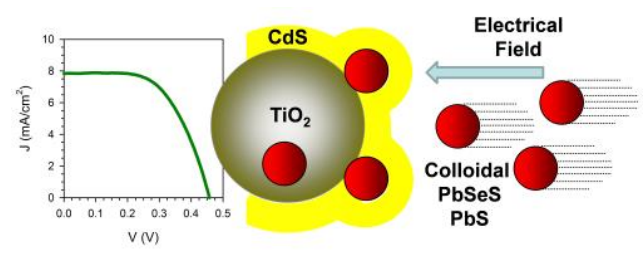

